九州大学学術情報リポジトリ

Kyushu University Institutional Repository

Reliability and Maintainability of a Multicomponent Series-Parallel System with Simultaneous Failure under Preemptive Repeat Repair Discipline

児玉，正憲

https://doi.org/10.15017/4491667

出版情報 : 經濟學研究. 52 (6)，pp.9-23，1987-08-10. 九州大学経済学会 バージョン：

権利関係 : 


\section{RELIABILITY AND MAINTAINABILITY OF \\ A MULTICOMPONENT SERIES-PARALLEL SYSTEM WITH SIMULTANEOUS FAILURE UNDER PREEMPTIVE REPEAT REPAIR DISCIPLINE}

\section{Masanori KODAMA}

\section{INTRODUCTION}

In this paper we consider a multicomponent system composed of two subsystem $S_{0}$ and $S_{1}$ in series. Subsystem $S_{0}$ is K-out-of-N : G system (more than $\mathrm{N}-\mathrm{K}+1$ must fail for $S_{0}$ to fail), while subsystem $S_{1}$ consists of $M$ different units connected in series (the failure of any one causes $S_{1}$ to fail).

The life-times of the active units depend on each other in having simultaneous failure of all the operating units and repair times are distributed quite generally. A failed unit is repaired at a single service channel. Kulshrestha [1,2] has studied the model for $K=1$ under the assumption that the repair of any unit is possible only when the system stops operating and the life times of the active units are mutually independent. Nakamichi et al. [3] and Kodama [4] have studied the model for $K=1$ with exponential failure distribution under the different repair policies. Kodama et al. [5] have investigated the system presented here under the assumption that the repair of any unit is possible only when the system stops operating. Assuming that the failed unit is repaired as soon as the unit fails and the system has priority in repair, Laplace transform of pointwise availability and reliability, mean time to the first system failure and steady state availability are derived. In practice, there are a lot of cases which the above conditions are satisfied (for example, an electronics and communication system, a space shuttle,....) and the above mentioned results are used for the reliability design of complex system, so it is important to study the above system. And also the theoretical interest in reliability analysis of the above system exists.

\section{DEFINITION OF MODELS AND NOTATIONS}

Independent Poisson processes $Z_{1}\left(t ; \lambda_{0}\right), \cdots, Z_{N}\left(t ; \lambda_{0}\right), Z_{1}\left(t ; \lambda_{1}\right), \cdots, Z_{M}\left(t ; \lambda_{M}\right)$ and $Z(t$; 
$\lambda^{*}$ ) govern the occurrence of shock (By $Z(t ; \lambda)$, we mean a Poisson process with a parameter $\lambda)$. Events in the process $Z_{i}\left(t ; \lambda_{0}\right)$ are shocks to unit $i$ only in $S_{0}(1 \leqq i \leqq N)$, and events in the process $Z_{j}\left(t ; \lambda_{j}\right)$ are shocks to unit $j$ only in $S_{1}(1 \leqq j \leqq M)$, and events in the process $Z\left(t ; \lambda^{*}\right)$ are shocks to all operating units.

Each unit results in failure after receiving only one "shock". The system breaks down if more than $(N-K+1)$ units in $S_{0}$ are simultaneously in a failed state or if any failure in $S_{1}$ occurs or if simultaneous failure occurs. A failed unit is repaired at a single service channel, and the repaired unit is assumed to behave like new after repair. Repair times are distributed quite generally. When the system is in a failed state, the residual good units do not fail.

While the system is operating, whenever any unit in $S_{0}$ fails, it is sent to the repair station at once where it is repaired on a first-come-first-served basis.

A repaired unit is put in operation immediately. When the system stops operating by stop of $S_{0}$, as soon as the unit in $S_{0}$ being served is repaired, it is reentered and then the system operates again. When the system stops operating by the stop of $S_{1}$, as soon as the failed unit of $S_{1}$ is repaired the system operates again. If a unit in $S_{1}$ fails when a unit in $S_{0}$ is being repaired, it is repaired without dely by interrupting the repair of unit from $S_{0}$; the preempted unit from $S_{0}$ repeat service from the begining on its reentry (preemptive repeat repair discipline). Through this model, $m$ units in $S_{0}(K \leqq m \leqq N)$ and all the units in $S_{1}$ are repaired when the system stops operating by the simultaneous failure. If the simultaneous failure occurs when a unit in $S_{0}$ is being repaired, all unit in $S_{1}$ and $m$ units in $S_{0}(K \leqq m \leqq N-1)$ are repaired without dely by interrupting the repair of the unit from $S_{0}$, if any ; the preempted unit from $S_{0}$ obeys the 'repeat rule' so that upon reentry its repair is to be started from the beginning. Similarly, we can discuss for the case of preemptive resumption priority (see reference [6]).

The system is always in one of the following states: $E_{N}$-all the unit in $S_{0}$ and $S_{1}$ are operative; $E_{i}-i$ units in $S_{0}$ and all the units in $S_{1}$ are operative, and a failed unit is undergoing repair, $K \leqq i \leqq N-1 ; F_{K-1}-(N-K+1)$ units in $S_{0}$ have failed and the system is undergoing repair ; $F_{i j}-i$ units in $S_{0}$ are operative and the system stops operating by the failure of the jth unit in $S_{1}$ and the system is undergoing repair, $K \leqq i \leqq N, 1 \leqq j \leqq M ; F_{0}$-all the units in $S_{0}$ and $S_{1}$ have failed and the system is undergoing repair. We note that $E$. means that the system is operating and $F$. means that the system is failed. Initially, all the units are operating.

Through this paper we use the following notations;

$$
\lambda=\sum_{j=1}^{M} \lambda_{j}
$$

$f_{0}(t)$ is the density function of repair time of a unit in $S_{0}$,

$$
f_{0}(t)=\mu_{0}(t) \exp \left[-\int_{0}^{t} \mu_{0}(x) d x\right]
$$


$f_{j}(t)$ is the density function of repair time of $j$ th unit in $S_{1}$,

$$
f_{j}(t)=\mu_{j}(t) \exp \left[-\int_{0}^{t} \mu_{j}(x) d x\right], \quad 1 \leqq j \leqq M, f(t)=\sum_{j=1}^{M} \lambda_{j} f_{j}(t) / \lambda ;
$$

$g_{i+M}^{*}(t)$ is the density function of repair time of $i$ units in $S_{0}$ and $M$ units in $S_{1}$ when the system stops operating by the simultaneous failure,

$$
g_{i+M}^{*}(t)=\mu_{i+M}^{*}(t) \exp \left[-\int_{o}^{t} u_{i+M}^{*}(x) d x\right], \quad K \leqq i \leqq N ;
$$

$K_{0}, K_{j}$ and $K_{i+M}^{*}$ are the first moment (mean repair time) of $f_{0}(t), f_{j}(t)$ and $g_{i+M}^{*}(t)$ respectively ; $\delta$ is the Kronecker delta ;

$\left(\begin{array}{c}m \\ i\end{array}\right)$ is the binomial coefficient that is assumed to be zero if $m<i ; P_{i}(t)=P$ \{the system is in state $E_{i}$ at time $\left.t\right\}, K \leqq i \leqq N$;

$P_{i}(t, x) d x=P$ \{the system is in state $E_{i}$ at time $t$ and elapsed repair time lies between $x$ and $x$ $+d x\}$

$$
P_{i}(t)=\int_{0}^{t} P_{i}(t, x) d x, \quad K \leqq i \leqq N-1 ;
$$

$P_{K-1}(t)=P$ the system is in state $F_{K-1}$ at time $t$ \}

$P_{K-1}(t, x) d t=P$ the system is in state $F_{K-1}$ at time $t$ and elapsed repair time lies between $x$ and $x+d x\}$,

$$
P_{K-1}(t)=\int_{0}^{t} P_{K-1}(t, x) d x ;
$$

$\mathrm{P}_{i j}(t)=P$ the system is in state $F_{i j}$ at time $\left.t\right\}$

$P_{i j}(t, x) d x=P$ the system is in state $F_{i j}$ at time $t$ and elapsed repair time lies between $x$ and $x+d x\}$

$$
P_{i j}(t)=\int_{0}^{t} P_{i j}(t, x) d x, \quad K \leqq i \leqq N, \quad 1 \leqq j \leqq N ;
$$

$Q_{0}(t)=P$ the system is in state $F_{0}$ at time $\left.t\right\}$

$Q_{0}(t, x) d t=P$ the system is in state $F_{0}$ at time $t$ and elapsed repair time lies between $x$ and $x$ $+d x\}$

$$
Q_{0}(t)=\int_{0}^{t} Q_{0}(t, x) d x
$$

$P_{A}(t)=P$ the system is functioning at time $\left.t\right\}=\sum_{i=k}^{N} P_{i}(t)$

(pointwise availability function)

$R(t)=P$ the system does not break down during $[0, t]\}$

(reliability function)

MTSF is the mean time to the first system failure $\bar{M}(s)$ is the Laplace transform of $M(t), \vec{M}(s)$ $=\int_{0}^{\infty} e^{-s t} M(t) d t$ 


$$
\begin{aligned}
& P_{i}(x)=\lim _{t \rightarrow \infty} P_{i}(t, x), \quad K-1 \leqq i \leqq N-1 ; \\
& P_{i}=\int_{0}^{\infty} P_{i}(x) d x, \quad K-1 \leqq i \leqq N-1 ; \\
& P_{N}=\lim _{t \rightarrow \infty} P_{N}(t) \text {; } \\
& P_{i j}(x)=\lim _{t \rightarrow \infty} P_{i j}(t, x), \quad K \leqq i \leqq N, \quad 1 \leqq j \leqq M \text {; } \\
& P_{j i}=\int_{0}^{\infty} P_{i j}(x) d x, \quad K \leqq i \leqq N, \quad 1 \leqq j \leqq M ; \\
& Q_{0}(x)=\lim _{t \rightarrow \infty} Q_{0}(t, x) \text {; } \\
& Q_{0}=\int_{0}^{\infty} Q_{0}(x) d x \text {; } \\
& P_{A}=\sum_{i=K}^{N} P_{i} \text { (steady state availability of the system); } \\
& \alpha\left(s, i, \lambda^{*}\right)=s+i \lambda_{0}+\lambda(1-f(s))+\lambda^{*}, \quad K \leqq i \leqq N \text {; } \\
& V_{i}^{(1)}(s)=\left\{\begin{array}{cc}
\prod_{r=K+1}^{i}\left\{l-\bar{f}_{0}\left(s+r \lambda_{0}+\lambda+\lambda^{*}\right)-\lambda \bar{f}(s)\left(1-\bar{f}_{0}\left(s+r \lambda_{0}+\lambda+\lambda^{*}\right)\right) /\right. \\
\left.\left.\left(s+r \lambda_{0}+\lambda+\lambda^{*}\right)\right] / \bar{f}_{0}\left(s+r \lambda_{0}+\lambda+\lambda^{*}\right)\right\}, & K+1 \leqq i \leqq N-1, \\
1, & i=K
\end{array},\right.
\end{aligned}
$$

$\sum_{n=i}^{j} h(n)=0, \quad i>j(h(n)$ is a function defined for $n=i, \cdots j)$

$$
\begin{aligned}
& C_{i, j}^{(1)}(s)=\sum_{n=i}^{j}\left(\begin{array}{c}
N \\
n
\end{array}\right) \alpha\left(s, n, \lambda^{*}\right) V_{n-1}^{(1)}(s) / \alpha\left(s, N, \lambda^{*}\right) ; \\
& E_{i, j}^{(1)}(s)=\sum_{n=i}^{j}\left(\begin{array}{c}
N \\
n
\end{array}\right)(N-n) \lambda_{0} V_{n-1}^{(1)}(s) / \alpha\left(s, N, \lambda^{*}\right) \text {; } \\
& E_{i, j}^{(1), m}=\sum_{n=i}^{j}\left(\begin{array}{c}
m \\
n
\end{array}\right) V_{n-1}^{(1)}(s) \text {; } \\
& a_{i}^{(1)}(s)=\bar{f}_{0}\left(s+K \lambda_{0}+\lambda+\lambda^{*}\right) C_{i+1, N}^{(1)}(s) /\left[\bar{f}_{0}\left(s+i \lambda_{0}+\lambda+\lambda^{*}\right) V_{i}^{(1)}(s) C_{k+1, N}^{(1)}(s)\right], \\
& b_{i}^{(1)}(s)=\left[C_{K+1, N}^{(1)}(s) E_{K+1, i}^{(1), m}(s)-C_{K-1, i}^{(1)}(s) E_{K+1, N-1}^{(1), m}(s)\right] / \\
& {\left[\bar{f}_{0}\left(s+i \lambda_{0}+\lambda+\lambda^{*}\right) V_{i}^{(1)}(s) C_{K+1, N}^{(1)}(s)\right], \quad K+1 \leqq i \leqq N-1} \\
& b_{i}^{(1) *}(s)=\left[C_{k+1, N}^{(1)}(s) E_{K+1, i}^{(1)}(s)-C_{K+1, i}^{(1)}(s) E_{K+1, N-1}^{(1)}(s)\right] / \\
& {\left[\bar{f}_{0}\left(s+i \lambda_{0}+\lambda+\lambda^{*}\right) V_{i}^{(1)}(s) C_{K+1, N}^{(1)}(s)\right], \quad K+1 \leqq i \leqq N-1} \\
& k_{1}^{(1)}(s)=\left\{1-\lambda^{*} \bar{g}_{m+M}^{*}(s) \bar{f}_{0}\left(s+(N-1) \lambda_{0}+\lambda+\lambda^{*}\right) b_{N-1}^{(1)}(s) /\right. \\
& \alpha\left(s, N, \lambda^{*}\right)-\lambda^{*} \bar{g}_{m+M}^{*}(s) \sum_{n=K}^{N-1}(-1)^{n-K}\left(\begin{array}{c}
n-1 \\
K-1
\end{array}\right)\left(1-\bar{f}_{0}\left(s+n \lambda_{0}+\lambda+\lambda^{*}\right)\right) \\
& \left.\cdot b_{n}^{(1)}(s) /\left(s+n \lambda_{0}+\lambda+\lambda^{*}\right)\right\}^{-1}\left\{\bar{f}_{0}\left(s+(N-1) \lambda_{0}+\lambda+\lambda^{*}\right) a_{N-1}^{(1)}(s) / \alpha\left(s, N, \lambda^{*}\right)\right. \\
& \left.+\sum_{n=K}^{N-1}(-1)^{n-K}\left(\begin{array}{c}
n-1 \\
K-1
\end{array}\right)\left(1-\bar{f}_{0}\left(s+n \lambda_{0}+\lambda+\lambda^{*}\right)\right) a_{n}^{(1)}(s) /\left(s+n \lambda_{0}+\lambda+\lambda^{*}\right)\right\} \\
& k_{0}^{(1)}(s)=\left\{1-\lambda^{*} \bar{g}_{m+M}^{*}(s) \bar{f}_{0}\left(s+(N-1) \lambda_{0}+\lambda+\lambda^{*}\right) b_{N-1}^{(1)}(s) /\right. \\
& \alpha\left(s, N, \lambda^{*}\right)-\lambda^{*} \bar{g}_{m+M}^{*}(s) \sum_{n=K}^{N-1}(-1)^{n-K}\left(\begin{array}{c}
n-1 \\
K-1
\end{array}\right)\left(1-\bar{f}_{0}\left(s+n \lambda_{0}+\lambda^{*}\right)\right. \\
& \left.\cdot b_{n}^{(1)}(s) /\left(s+n \lambda_{0}+\lambda+\lambda^{*}\right)\right\}^{-1}\left\{\left[1+\bar{f}_{0}\left(s+(N-1) \lambda_{0}+\lambda+\lambda^{*}\right) b_{N-1}^{(1) *}(s)\right] /\right. \\
& \alpha\left(s, N, \lambda^{*}\right)+\sum_{n=K}^{N-1}(-1)^{n-K}\left(\begin{array}{c}
n-1 \\
K-1
\end{array}\right)\left(1-\bar{f}_{0}\left(s+n \lambda_{0}+\lambda+\lambda^{*}\right)\right) b_{n}^{(1) *}(s) / \\
& \left.\left(s+n \lambda_{0}+\lambda+\lambda^{*}\right)\right\} \\
& a_{i}^{(1)}(s)=a_{i}^{(1)}(s)+\lambda^{*} \bar{g}_{m+M}^{*}(s) k_{1}^{(1)}(s) b_{i}^{(1)}(s), \quad K+1 \leqq i \leqq N-1 \text {; } \\
& b_{i}^{(1)}(s)=\lambda^{*} \bar{g}_{m+M}^{*}(s) k_{0}^{(1)}(s) b_{i}^{(1)}(s)+b_{i}^{(1) *}(s), \quad K+1 \leqq i \leqq N-1 \text {; }
\end{aligned}
$$




\section{RELIABILITY AND MAINTAINABILITY OF A MULTICOMPONENT SERIES-PARALLEL SYSTEM}

\section{SOLUTIONS OF THE PROBLEM}

The analyses are classified to according to the nature of this system and the relations among $m, K$, and $N$ and in this way three cases may occur : i) $1 \leqq K \leqq m \leqq N-1$, ii) $1 \leqq K \leqq N-1, m=$ $N$, iii) $2 \leqq K=N, m=N$. The unified analysis of three cases is more difficult than a separate analysis. Therefore, we make the separate analysis of three cases. The analysis crucially depends on the method of supplementary variables, and the supplementary variable $x$ denotes the elapsed time that a unit has been undergoing repair. Viewing the nature of this model, we obtain the following set of differential-difference equations :

(i) $1 \leqq K \leqq m \leqq N-1$,

$$
\begin{aligned}
& {\left[d / d t+N \lambda_{0}+\lambda+\lambda^{*}\right] P_{N}(t)=\int_{0}^{t} \mu_{0}(x) P_{N-1}(t, x) d x+\sum_{j=1}^{M} \int_{0}^{t} \mu_{j}(x) P_{N j}(t, x) d x, } \\
& {\left[\partial / \partial t+\partial / \partial x+i \lambda_{0}+\lambda+\lambda^{*}+\mu_{0}(x)\right] P_{i}(t, x)=\left(1-\delta_{i, N-1}\right)(i+1) \lambda_{0} P_{i+1}(t, x), \quad K \leqq i \leqq N-1, } \\
& {\left[\partial / \partial t+\partial / \partial x+\mu_{0}(x)\right] P_{K-1}(t, x)=K \lambda_{0} P_{K}(t, x), } \\
& {\left[\partial / \partial t+\partial / \partial x+\mu_{j}(x)\right] P_{i j}(t, x)=0, \quad K \leqq i \leqq N, \quad 1 \leqq j \leqq M, } \\
& {\left[\partial / \partial t+\partial / \partial x+\mu_{m+M}^{*}(x)\right] Q_{0}(t, x)=0, } \\
& P_{i}(t, 0)=\delta_{i, N-1} N \lambda_{0} P_{N}(t)+\int_{0}^{t} \mu_{0}(x) P_{i-1}(t, x) d x+\sum_{j=1}^{M} \int_{0}^{t} \mu_{j}(x) P_{i j}(t, x) d x \\
& \quad+\delta_{i, m} \int_{0}^{t} \mu_{m+M}^{*}(x) Q_{0}(t, x) d x, \quad K \leqq i \leqq N-1, \\
& P_{K-1}(t, o)= \\
& P_{i j}(t, 0)= \\
& Q_{o}(t, 0)= \lambda_{j} P_{i=K}^{N} P_{i}(t), \quad K \leqq i \leqq N, \quad 1 \leqq j \leqq M, \\
&= \lambda^{*} P_{N}(t)+\lambda^{*} \sum_{i=K}^{N-1} \int_{0}^{t} P_{i}(t, x) d x, \\
& P_{N}(0)= 1 .
\end{aligned}
$$

In order to solve these equations we introduce the discrete transform (see reference [10], p. 26-30).

$$
\begin{aligned}
U_{i}(t, x) & =\sum_{n=i}^{N-1}\left(\begin{array}{c}
n \\
i
\end{array}\right) P_{n}(t, x), \quad K \leqq i \leqq N-1, \\
P_{i}(t, x) & =\sum_{n=i}^{N-1}(-1)^{n-i}\left(\begin{array}{c}
n \\
i
\end{array}\right) U_{n}(t, x), \quad K \leqq i \leqq N-1
\end{aligned}
$$

Taking the Laplace transform of (3.1)-(3.9), (3.11) and (3.12) under the initial condition and using the results of Appendix 1, we obtain.

$$
\begin{aligned}
& \bar{P}_{i j}(s)=\lambda_{j} \bar{P}_{i}(s)\left(1-\bar{f}_{j}(s)\right) / s, \quad K \leqq i \leqq N, \quad 1 \leqq j \leqq M, \\
& \bar{Q}_{0}(s)=\lambda^{*} \sum_{i=K}^{N} \bar{P}_{i}(s)\left(1-\bar{g}_{m+M}^{*}(s)\right) / s, \\
& \bar{P}_{i}(s)=\sum_{n=i}^{N-1}(-1)^{n-i}\left(\begin{array}{c}
n \\
i
\end{array}\right) \bar{U}_{n}(s, 0)\left[1-\bar{f}_{0}\left(s+n \lambda_{0}+\lambda+\lambda^{*}\right)\right] /\left(s+n \lambda_{0}+\lambda+\lambda^{*}\right), \\
& \bar{P}_{N}(s)=\left[\bar{f}_{0}\left(s+(N-1) \lambda_{0}+\lambda+\lambda^{*}\right) \bar{U}_{N-1}(s, 0)+1\right] / \alpha\left(s, N, \lambda^{*}\right),
\end{aligned}
$$




$$
\begin{aligned}
\bar{P}_{K-1}(s)= & K \lambda_{0} \sum_{n=K}^{N-1}(-1)^{n-K}\left(\begin{array}{c}
n \\
K
\end{array}\right)\left[\bar{U}_{n}(s, 0) /\left(n \lambda_{0}+\lambda+\lambda^{*}\right)\right] \\
& \cdot\left[\left(1-\bar{f}_{0}(s)\right) / s-\left(1-\bar{f}_{0}\left(s+n \lambda_{0}+\lambda+\lambda^{*}\right)\right) /\left(s+n \lambda_{0}+\lambda+\lambda^{*}\right)\right] \\
\bar{U}_{i}(s, 0)= & a_{i}^{(1) \prime}(s) \bar{U}_{K}(s, 0)+b_{i}^{(1) \prime}(s), \quad K+1 \leqq i \leqq N-1 \\
\bar{U}_{K}(s, 0)= & \left(\begin{array}{l}
N \\
K
\end{array}\right)\left[(N-K) \lambda_{0}-\alpha\left(s, K, \lambda^{*}\right) \bar{f}_{0}\left(s+(N-1) \lambda_{0}+\lambda+\lambda^{*}\right) b_{N-1}^{(1)}(s)\right] / \alpha\left(s, N, \lambda^{*}\right) \\
& +\sum_{n=K}^{N-1}(-1)^{n-K}\left[\left(\begin{array}{c}
n \\
K-1
\end{array}\right) \bar{f}_{0}\left(s+n \lambda_{0}+\lambda+\lambda^{*}\right)+\left(\begin{array}{c}
n \\
K
\end{array}\right) K \lambda_{0}\left(\bar{f}_{0}(s)-\bar{f}_{0}\left(s+n \lambda_{0}+\lambda+\lambda^{*}\right)\right) /\right. \\
& \left.\left.\left(n \lambda_{0}+\lambda+\lambda^{*}\right)\right] b_{n}^{(1) \prime}(s)+\left(\begin{array}{l}
m \\
K
\end{array}\right) \lambda^{*} \bar{g}_{m+M}^{*}(s) k_{0}^{(1)}(s)\right\} \\
& \cdot\left\{1-\bar{f}_{0}\left(s+K \lambda_{0}+\lambda+\lambda^{*}\right)-\lambda \bar{f}^{\prime}(s)\left[1-\bar{f}_{0}\left(s+K \lambda_{0}+\lambda+\lambda^{*}\right)\right] /\left(s+K \lambda_{0}+\lambda+\lambda^{*}\right)\right. \\
& +\left(\begin{array}{c}
N \\
K
\end{array}\right) \alpha\left(s, K, \lambda^{*}\right) \bar{f}_{0}\left(s+(N-1) \lambda_{0}+\lambda+\lambda^{*}\right) a N_{-1}^{(1)}(s) / \\
& \alpha\left(s, N, \lambda^{*}\right)-\sum_{n=K}^{N-1}(-1)^{n-K}\left[\left(\begin{array}{c}
n \\
K-1
\end{array}\right) \bar{f}_{0}\left(s+n \lambda_{0}+\lambda+\lambda^{*}\right)+\left(\begin{array}{c}
n \\
K
\end{array}\right) K \lambda_{0}\left(\bar{f}_{0}(s)\right.\right. \\
& \left.-\bar{f}_{0}\left(s+n \lambda_{0}+\lambda+\lambda^{*}\right)\right) / \\
& \left.\left.\left(n \lambda_{0}+\lambda+\lambda^{*}\right)\right] a_{n}^{(1) \prime}(s)-\left(\begin{array}{l}
m \\
K
\end{array}\right) K \lambda^{*} \bar{g}_{m+M}(s) k_{1}^{(1)}(s)\right\} \\
\sum_{j=K}^{N} \bar{P}_{j}(s)= & k_{1}^{(1)}(s) \bar{U}_{K}(s, 0)+k_{0}^{(1)}(s),
\end{aligned}
$$

$P_{i j}(t), Q_{0}(t), P_{K-1}(t)$ and $P_{i}(t)$ are the probability that the system are in state $F_{i j}, F_{0}, F_{K-1}$ and $E_{i}$ at time $t$ respectively. Hence the Laplace transform of the system availability $\bar{P}_{A}(s)$ is given by (3.20).

The Laplace transform of system reliability $\bar{R}(s)$ can be obtain from $\bar{P}_{A}(s)$ by modifying the system so that a down state is an absorbing state, it can never go up. So we substitute $\bar{f}(s)=$ $0,\left(\bar{f}_{0}(s)-\bar{f}_{0}\left(s+n \lambda_{0}+\lambda+\lambda^{*}\right)\right)=0, K \leqq n \leqq N-1$ and $\bar{g}_{m+M}^{*}(s)=0$ in $\bar{P}_{A}(s)$. Moreover, if we set $s$ $=0$ in $\bar{R}(s)$, we obtain MTSF.

In order to derive the steady state availability we set up the following set of differentialdifference equations :

$$
\begin{aligned}
& {\left[N \lambda_{0}+\lambda+\lambda^{*}\right] P_{N}=\int_{0}^{\infty} \mu_{0}(x) P_{N-1}(x) d x+\sum_{j=1}^{M} \int_{0}^{\infty} \mu_{j}(x) P_{N_{j}}(x) d x,} \\
& {\left[d / d x+i \lambda_{0}+\lambda+\lambda^{*}+\mu_{0}(x)\right] P_{i}(x)=\left(1-\delta_{i, N-1}\right)(i+1) \lambda_{0} P_{i+1}(x), \quad K \leqq i \leqq N-1,} \\
& {\left[d / d x+\mu_{0}(x)\right] P_{K-1}(x)=K \lambda_{0} P_{K}(x),} \\
& {\left[d / d x+\mu_{j}(x)\right] P_{i j}(x)=0, \quad K \leqq i \leqq N, \quad 1 \leqq j \leqq M,} \\
& {\left[d / d x+\mu_{m+M}^{*}(x)\right] Q_{0}(x)=0,}
\end{aligned}
$$

With the following boundary conditions and normalizing condition :

$$
\begin{aligned}
P_{i}(0)= & \delta_{i, N-1} N \lambda_{0} P_{N}+\int_{o}^{\infty} \mu_{0}(x) P_{i-1}(x) d x+\sum_{j=1}^{M} \int_{0}^{\infty} \mu_{j}(x) P_{i j}(x) d x \\
& +\delta_{i, m} \int_{0}^{\infty} \mu_{m+M}^{*}(x) Q_{0}(x) d x, \quad K \leqq i \leqq N-1, \\
P_{K-1}(0)= & 0, \\
P_{N j}(0)= & \lambda_{j} P_{N}, \quad 1 \leqq j \leqq M,
\end{aligned}
$$


RELIABILITY AND MAINTAINABILITY OF A MULTICOMPONENT SERIES-PARALLEL SYSTEM

$$
\begin{aligned}
& P_{i j}(0)=\lambda_{j} \int_{0}^{\infty} P_{i}(x) d x=\lambda_{j} P_{i}, \quad K \leqq i \leqq N-1, \quad 1 \leqq j \leqq M, \\
& Q_{0}(0)=\lambda^{*} P_{N}+\lambda^{*} \sum_{i=K}^{N-1} \int_{0}^{\infty} P_{i}(x) d x=\lambda^{*} \sum_{i=K}^{N} P_{i}, \\
& P_{N}+\sum_{i=K}^{N-1} \int_{0}^{\infty} P_{i}(x) d x+\int_{0}^{\infty} P_{K-1}(x) d x+\sum_{j=1}^{M} \int_{0}^{\infty} P_{N j}(x) d x+\sum_{i=K}^{N-1} \sum_{j=1}^{M} \int_{0}^{\infty} P_{i j}(x) d x \\
& \quad+\int_{0}^{\infty} Q_{0}(x) d x=1 .
\end{aligned}
$$

Solving the above equations, we obtain (see Appendix 2)

$$
\begin{aligned}
P_{i j}= & \lambda_{j} K_{j} P_{i}, \\
Q_{0}= & \lambda^{*} K^{*}{ }_{m+M} \sum_{i=K}^{N} P_{i} \\
P_{i}= & \sum_{n=i}^{N-1}(-1)^{n-i}\left(\begin{array}{c}
n \\
i
\end{array}\right) U_{n}(0)\left(1-\bar{f}_{0}\left(n \lambda_{0}+\lambda+\lambda^{*}\right)\right) /\left(n \lambda_{0}+\lambda+\lambda^{*}\right), \quad K \leqq i \leqq N-1 \\
P_{N}= & \bar{f}_{0}\left((N-1) \lambda_{0}+\lambda+\lambda^{*}\right) U_{N-1}(0) /\left(N \lambda_{0}+\lambda^{*}\right), \\
P_{K-1}= & \sum_{n=K}^{N-1}(-1)^{n-K}\left(\begin{array}{c}
n \\
K
\end{array}\right) K \lambda_{0} U_{n}(0)\left[K_{0}-\left(1-\bar{f}_{0}\left(n \lambda_{0}+\lambda+\lambda^{*}\right)\right) /\left(n \lambda_{0}+\lambda+\lambda^{*}\right)\right] /\left(n \lambda_{0}+\lambda+\lambda^{*}\right) \\
U_{i}(0)= & a_{i}^{(1) \prime}(0) U_{K}(0), \quad K+1 \leqq i \leqq N-1, \\
U_{K}(0)= & \left\{k_{1}^{(1)}(0)\left[1+\lambda^{*} K^{*}{ }_{m+M}+\sum_{j=1}^{M} \lambda_{j} K_{j}\right]+\sum_{n=K}^{N-1}(-1)^{n+K}\left(\begin{array}{l}
n \\
K
\end{array}\right)\left[K \lambda_{0} /\left(n \lambda_{0}+\lambda+\lambda^{*}\right)\right]\right. \\
& \left.\cdot\left[K_{0}-\left(1-\bar{f}_{0}\left(n \lambda_{0}+\lambda+\lambda^{*}\right)\right) /\left(n \lambda_{0}+\lambda+\lambda^{*}\right)\right] a_{n}^{(1) \prime}(0)\right\}^{-1} \\
\sum_{n=K}^{N} P_{n}= & k_{1}^{(1)}(0) U_{K}(0) .
\end{aligned}
$$

$P_{i j}, Q_{0}, P_{K-1}$ and $P_{i}$ are the probability that the system are in state $F_{i j}, F_{o}, F_{K-1}$, and $E_{i}$ in equilibrium respectively. Hence the stationary availability $P_{A}$ is given by (3.39) explicitly in closed form.

Remark 1) $\lambda^{*}=0$, we have from (3.38) and (3.39)

$$
\begin{aligned}
U_{K}(0)= & \left\{( 1 + \sum _ { j = 1 } ^ { M } \lambda _ { j } K _ { j } ) \left[\bar{f}_{0}\left((N-1) \lambda_{0}+\lambda\right) a_{N-1}^{(1)}(0) / N \lambda_{0}\right.\right. \\
& \left.+\sum_{n=K}^{N-1}(-1)^{n-K}\left(\begin{array}{c}
n-1 \\
K-1
\end{array}\right)\left(1-\bar{f}_{0}\left(n \lambda_{0}+\lambda\right)\right) a_{n}^{(1)}(0) /\left(n \lambda_{0}+\lambda\right)\right] \\
& +\sum_{n=K}^{N-1}(-1)^{n-K} K \lambda_{0}\left(\begin{array}{c}
n \\
K
\end{array}\right)\left[K_{0}-\left(1-\bar{f}\left(n \lambda_{0}+\lambda\right)\right)\left(n \lambda_{0}+\lambda\right)\right] a_{n}^{(1)}(0) / \\
& \left.\left(n \lambda_{0}+\lambda\right)\right\}^{-1}
\end{aligned}
$$

Equation (3.40) coincides with the formula (30)' of model 5 in M. Kodama [8].

Remark 2) $\lambda=0$, we have from (3.38) and (3.39)

$$
\begin{aligned}
P_{A}= & U_{K}(0)\left\{\bar{f}_{0}\left((N-1) \lambda_{0}+\lambda+\lambda^{*}\right) a_{N-1}^{(1)}(0) /\left(N \lambda+\lambda^{*}\right)\right. \\
& \left.+\sum_{n=K}^{N-1}(-1)^{n-K}\left(\begin{array}{c}
n-1 \\
K-1
\end{array}\right)\left[\left(1-\bar{f}_{0}\left(n \lambda_{0}+\lambda^{*}\right)\right) /\left(n \lambda_{0}+\lambda^{*}\right)\right] a_{n}^{(1) \prime}(0)\right\},
\end{aligned}
$$

where

$$
U_{K}(0)=\left\{\left(1+\lambda^{*} K_{m+M}^{*}\right) \bar{f}_{0}\left((N-1) \lambda_{0}+\lambda^{*}\right) a_{N-1}^{(1)}(0) /\left(N \lambda_{0}+\lambda^{*}\right)\right.
$$




$$
\begin{aligned}
& +\sum_{n=K}^{N-1}(-1)^{n-K}\left(\begin{array}{c}
n-1 \\
K-1
\end{array}\right) a_{n}^{(1) \prime}(0) \\
& \left.\cdot\left[n \lambda_{0} K_{0} /\left(n \lambda_{0}+\lambda^{*}\right)+\lambda^{*}\left(1-\bar{f}_{0}\left(n \lambda_{0}+\lambda^{*}\right)\right)\left(K_{m+M}^{*}+1 /\left(n \lambda_{0}+\lambda^{*}\right)\right) /\left(n \lambda_{0}+\lambda^{*}\right)\right]\right\}^{-1} .
\end{aligned}
$$

Equations (3.41) and (3.42) coincide with the formulas (29) and (28) in M. Kodama et. al [9], respectively.

(ii) $1 \leqq K \leqq N-1, m=N$

(3.2), (3.3), (3.4), (3.7), (3.8), (3.9) and (3.10) remain the same. Three new equations are added.

$$
\begin{aligned}
& {\left[d / d t+N \lambda_{0}+\lambda+\lambda^{*}\right] P_{N}(t)=} \int_{0}^{t} \mu_{0}(x) P_{N-1}(t, x) d x+\sum_{j=1}^{M} \int_{0}^{t} \mu_{j}(x) P_{N j}(t, x) d x \\
&+\int_{0}^{t} \mu_{N+M}^{*}(x) Q_{0}(t, x) d x, \\
& {\left[\partial / \partial t+\partial / \partial x+\mu_{N+M}^{*}(x)\right] Q_{0}(t, x)=0, } \\
& P_{i}(t, 0)=\delta_{i, N-1} N \lambda_{0} P_{N}(t)+\int_{0}^{t} \mu_{0}(x) P_{i-1}(t, x) d x+\sum_{j=1}^{M} \int_{0}^{t} \mu_{i}(x) P_{i j}(t, x) d x,
\end{aligned}
$$

By the similar method we obtain

$$
\begin{aligned}
& \bar{P}_{N}(s)=\left[\bar{f}_{0}\left(s+(N-1) \lambda_{0}+\lambda+\lambda^{*}\right) U_{N-1}(s, 0)+1+\lambda^{*} \bar{g}_{N+M}^{*}(s) \sum_{i=K}^{N} \bar{P}_{i}(s)\right] / \alpha\left(s, N, \lambda^{*}\right), \\
& \bar{P}_{i}(s)=\sum_{n=i}^{N-1}(-1)^{n-i}\left(\begin{array}{c}
n \\
i
\end{array}\right) U_{n}(s, 0)\left[1-\bar{f}_{0}\left(s+n \lambda_{0}+\lambda+\lambda^{*}\right)\right] /\left(s+n \lambda_{0}+\lambda+\lambda^{*}\right), \\
& 1 \leqq i \leqq N-1 \\
& U_{n}(s, 0)=a_{n}^{(2) \prime}(s) U_{K}(s, 0)+b_{n}^{(2) * *^{\prime}}(s), \quad K+1 \leqq n \leqq N-1, \\
& \bar{P}_{A}(s)=\sum_{i=K}^{N} \bar{P}_{i}(s)=k_{1}^{(2)}(s) U_{K}(s, 0)+k_{0}^{(2)}(s) \text {, } \\
& U_{K}(s, 0)=\left\{( \begin{array} { l } 
{ N } \\
{ K }
\end{array} ) \left[(N-K) N \lambda_{0}\left(1+\lambda^{*} \bar{g}_{N+M}^{*}(s) k_{0}^{(2)}(s)\right)-\alpha\left(s, K, \lambda^{*}\right)\right.\right. \\
& \left.\cdot \bar{f}\left(s+(N-1) \lambda_{0}+\lambda+\lambda^{*}\right) b_{N-1}^{(1) * \prime}(s)\right] / \alpha\left(s, N, \lambda^{*}\right) \\
& +\sum_{n=K}^{N-1}(-1)^{n-K}\left[\left(\begin{array}{c}
n \\
K-1
\end{array}\right) \bar{f}_{0}\left(s+n \lambda_{0}+\lambda+\lambda^{*}\right)+\left(\begin{array}{c}
n \\
K
\end{array}\right) K \lambda_{0}\left(\bar{f}_{0}(s)\right.\right. \\
& \left.\left.\left.-\bar{f}_{0}\left(s+n \lambda_{0}+\lambda+\lambda^{*}\right)\right) /\left(s+n \lambda_{0}+\lambda+\lambda^{*}\right)\right] b_{n}^{(1) *^{\prime}}(s)\right\}\left\{1-\bar{f}_{0}\left(s+K \lambda_{0}+\lambda+\lambda^{*}\right)\right. \\
& -\lambda \bar{f}(s)\left(1-\bar{f}_{0}\left(s+K \lambda_{0}+\lambda+\lambda^{*}\right)\right) /\left(s+K \lambda_{0}+\lambda+\lambda^{*}\right)+\left(\begin{array}{l}
N \\
K
\end{array}\right)\left[\alpha\left(s, K, \lambda^{*}\right)\right. \\
& \left.\cdot \bar{f}_{0}\left(s+(N-1) \lambda_{0}+\lambda+\lambda^{*}\right) a_{N-1}^{(1) \prime}(s)-(N-K) \lambda_{0} \lambda^{*} \bar{g}_{N+M}^{*}(s) k_{1}^{(2)}(s)\right] \\
& / \alpha\left(s, N, \lambda^{*}\right)-\sum_{n=K}^{N-1}(-1)^{n-K}\left[\left(\begin{array}{c}
n \\
K-1
\end{array}\right) \bar{f}_{0}\left(s+n \lambda_{0}+\lambda+\lambda^{*}\right)+\left(\begin{array}{c}
n \\
K
\end{array}\right) K \lambda_{0}\right. \\
& \left.\left.\cdot\left(\bar{f}_{0}(s)-\bar{f}_{0}\left(s+n \lambda_{0}+\lambda+\lambda^{*}\right)\right) /\left(s+n \lambda_{0}+\lambda+\lambda^{*}\right)\right] a_{n}^{(1) \prime}(s)\right\}^{-1} \text {, } \\
& a_{n}^{(2) \prime}(s)=a_{n}^{(1)}(s)+\lambda^{*} \bar{g}_{N+M}^{*}(s) k_{1}^{(2)}(s) b_{n}^{(1) *}(s), \quad K+1 \leqq n \leqq N-1, \\
& b_{n}^{(2) * \prime}(s)=\left(1+\lambda^{*} \bar{g}_{N+M}^{*}(s) k_{0}^{(2)}(s)\right) b_{n}^{(1) *}(s), \quad K+1 \leqq n \leqq N-1 \text {, } \\
& k_{1}^{(2)}(s)=\left\{1-\lambda^{*} \bar{g}_{N+M}^{*}(s)\left[1+\bar{f}_{0}\left(s+(N-1) \lambda_{0}+\lambda+\lambda^{*}\right) b_{N-1}^{(1) *}(s)\right] / \alpha\left(s, N, \lambda^{*}\right)\right. \\
& -\lambda^{*} \bar{g}_{N+M}^{*}(s) \sum_{n=K}^{N-1}(-1)^{n-K}\left(\begin{array}{c}
n-1 \\
K-1
\end{array}\right)\left[\left(1-\bar{f}_{0}\left(s+n \lambda_{0}+\lambda+\lambda^{*}\right)\right) /\left(s+n \lambda_{0}+\lambda+\lambda^{*}\right)\right]
\end{aligned}
$$


RELIABILITY AND MAINTAINABILITY OF A MULTICOMPONENT SERIES-PARALLEL SYSTEM

$$
\begin{aligned}
& \left.\cdot b_{n}^{(1) *}(s)\right\}^{-1}\left\{\bar{f}_{0}\left(s+(N-1) \lambda_{0}+\lambda+\lambda^{*}\right) a_{N-1}^{(1)}(s) / \alpha\left(s, N, \lambda^{*}\right)\right. \\
& \left.+\sum_{n=K}^{N-1}(-1)^{n-K}\left(\begin{array}{c}
n-1 \\
K-1
\end{array}\right)\left[\left(1-\bar{f}_{0}\left(s+n \lambda_{0}+\lambda+\lambda^{*}\right)\right) /\left(s+n \lambda_{0}+\lambda+\lambda^{*}\right)\right] a_{n}^{(1)}(s)\right\},
\end{aligned}
$$

$$
\begin{aligned}
k_{0}^{(2)}(s)= & \left\{1-\lambda^{*} \bar{g}_{N+M}^{*}(s)\left[1+\bar{f}_{0}\left(s+(N-1) \lambda_{0}+\lambda+\lambda^{*}\right) b_{N-1}^{(1) *}(s)\right] / \alpha\left(s, N, \lambda^{*}\right)\right. \\
& -\lambda^{*} \bar{g}_{N+M}^{*}(s) \sum_{n=K}^{N-1}(-1)^{n-K}\left(\begin{array}{c}
n-1 \\
K-1
\end{array}\right)\left[\left(1-\bar{f}_{0}\left(s+n \lambda_{0}+\lambda+\lambda^{*}\right)\right) /\left(s+n \lambda_{0}+\lambda+\lambda^{*}\right)\right] \\
& \left.\cdot b_{n}^{(1) *}(s)\right\}^{-1}\left\{\left[1+f_{0}\left(s+(N-1) \lambda_{0}+\lambda+\lambda^{*}\right) b_{N-1}^{(1) *}(s)\right] / \alpha\left(S, N, \lambda^{*}\right)\right. \\
& \left.+\sum_{n=K}^{N-1}(-1)^{n-K}\left(\begin{array}{c}
n-1 \\
K-1
\end{array}\right)\left[\left(1-\bar{f}_{0}\left(s+n \lambda_{0}+\lambda+\lambda^{*}\right)\right) /\left(s+n \lambda_{0}+\lambda+\lambda^{*}\right)\right] b_{n}^{(1) *}(s)\right\} .
\end{aligned}
$$

Stationary availability (3.22)-(3.24), (3.27)-(3.31) remain the same. Three new equations are added :

$$
\begin{aligned}
{\left[N \lambda_{0}+\lambda+\lambda^{*}\right] P_{N}=} & \int_{0}^{\infty} \mu_{0}(x) P_{N-1}(x) d x+\sum_{j=1}^{M} \int_{0}^{\infty} \mu_{j}(x) P_{N j}(x) d x \\
& +\int_{0}^{\infty} \mu_{N+M}^{*}(x) Q_{0}(x) d x,
\end{aligned}
$$

$\left[d / d x+\mu_{N+M}^{*}(x)\right] Q_{0}(x)=0$ ，

$$
P_{i}(0)=\delta_{i, N-1} N \lambda_{0} P_{N}+\int_{0}^{\infty} \mu_{0}(x) P_{i-1}(x) d x+\sum_{j=1}^{M} \int_{0}^{\infty} \mu_{j}(x) P_{i j}(x) d x .
$$

By the similar method we obtain

$$
\begin{aligned}
P_{N}= & \left\{\bar{f}_{0}\left((N-1) \lambda_{0}+\lambda+\lambda^{*}\right) U_{N-1}(0)+\lambda^{*} \sum_{i=K}^{N} P_{i}\right\} /\left(N \lambda_{0}+\lambda^{*}\right), \\
P_{i}= & \sum_{n=i}^{N-1}(-1)^{n-i}\left(\begin{array}{c}
n \\
i
\end{array}\right) U_{i}(0)\left(1-f_{0}\left(i \lambda_{0}+\lambda+\lambda^{*}\right)\right) /\left(i \lambda_{0}+\lambda+\lambda^{*}\right), \quad K \leqq i \leqq N-1, \\
P_{K-1}= & \sum_{n=K}^{N}(-1)^{n-K} \\
& \left(\begin{array}{l}
n \\
K
\end{array}\right) U_{n}(0)\left[K \lambda_{0} /\left(n \lambda_{0}+\lambda+\lambda^{*}\right)\right]\left[K_{0}-\left(1-\bar{f}_{0}\left(n \lambda_{0}+\lambda+\lambda^{*}\right)\right) /\left(n \lambda_{0}+\lambda+\lambda^{*}\right)\right], \\
U_{i}(0)= & a_{i}^{(2)}(0) U_{K}(0), \\
P_{i j}= & \lambda_{j} K_{j} P_{i}, K \leqq i \leqq N, \quad K \leqq i \leqq M, \\
Q_{0}= & \lambda^{*} K_{N+M}^{*} \sum_{i=K}^{N} P_{i}=\lambda^{*} K_{N+M}^{*} P_{A}, \\
P_{A}= & \sum_{i=K}^{N} P_{i}=k_{1}^{(2)}(0) U_{K}(0), \\
a_{i}^{(2) \prime}(0)= & a_{i}^{(1)}(0)+\lambda^{*} k_{1}^{(2)}(0) b_{i}^{(1) *}(0), \\
U_{K}(0)= & \left\{k_{1}^{(2)}(0)\left[1+\lambda^{*} K_{N+M}^{*}+\sum_{j=1}^{M} \lambda_{j} K_{j}\right]+\sum_{n=K}^{N-1}(-1)^{n-K}\left(\begin{array}{l}
n \\
K
\end{array}\right)\left[K \lambda_{0} /\left(n \lambda_{0}+\lambda+\lambda^{*}\right)\right]\right. \\
& \left.\left.\cdot K_{0}-\left(1-\bar{f}_{0}\left(n \lambda+\lambda+\lambda^{*}\right)\right) /\left(n \lambda_{0}+\lambda+\lambda^{*}\right)\right] a_{n}^{(1) \prime}(0)\right\}^{-1},
\end{aligned}
$$

(iii) $2 \leqq K=N, m=N \quad$ (3.10), (3.43) and (3.44) remain the same. Five new equations are added:

$\left[\partial / \partial t+\partial / \partial x+\mu_{0}(x)\right] P_{K-1}(t, x)=0$,

$\left[\partial / \partial t+\partial / \partial x+\mu_{j}(x)\right] P_{N j}(t, x)=0, \quad 1 \leqq j \leqq M$,

$P_{N j}(t, 0)=\lambda_{j} P_{N}(t), \quad 1 \leqq j \leqq M$, 


$$
\begin{aligned}
P_{K-1}(t) & =N \lambda_{0} P_{N}(t), \\
Q_{0}(t, 0) & =\lambda^{*} P_{N}(t) .
\end{aligned}
$$

The Laplace transform of $P_{A}(t)$ and $R(t)$, MTSF and $P_{A}$ are derived easily.

$$
\begin{aligned}
\bar{P}_{A}(s) & =\bar{P}_{N}(s)=\left\{s+N \lambda_{0}\left(1-\bar{f}_{0}(s)\right)+\lambda(1-\bar{f}(s))+\lambda^{*}\left(1-\bar{g}_{N+M}^{*}(s)\right)\right\}^{-1}, \\
\bar{P}_{K-1}(s) & =N \lambda_{0} \bar{P}_{N}(s)(1-\bar{f}(s)) / s, \\
\bar{P}_{N j}(s) & =\lambda_{j} \bar{P}_{N}(s)\left(1-\bar{f}_{j}(s)\right) / s, \quad 1 \leqq j \leqq M, \\
\bar{Q}_{0}(s) & =\lambda^{*} \bar{P}_{N}(s)\left(1-\bar{g}_{N+M}^{*}(s)\right) / s, \\
P_{A} & =P_{N}=\left(1+N \lambda_{0} K_{0}+\sum_{j=1}^{M} \lambda_{j} K_{j}+\lambda^{*} K_{N+M}^{*}\right)^{-1}, \\
P_{K-1} & =N \lambda_{0} K_{0} P_{N}, \\
P_{N j} & =\lambda_{j} K_{j} P_{N}, \quad 1 \leqq j \leqq M, \quad Q_{0}=\lambda^{*} K_{N+M}^{*} P_{N}
\end{aligned}
$$

\section{APPENDIXES}

\section{Appendix 1}

Taking the Laplace transform of (1.1)-(1.9) under the initial condition (3.10), we have

$$
\begin{aligned}
& {\left[s+N \lambda_{0}+\lambda+\lambda^{*}\right] \bar{P}_{N}(s)=\int_{0}^{\infty} \mu_{0}(x) \bar{P}_{N-1}(s, x) d x+\sum_{j=1}^{M} \int_{0}^{\infty} \mu_{j}(x) \bar{P}_{N j}(s, x) d x,} \\
& {\left[s+\partial / \partial x+i \lambda_{0}+\lambda+\lambda^{*}+\mu_{0}(x)\right] \bar{P}_{i}(s, x)=\left(1-\delta_{i, N-1}\right)(i+1) \lambda_{0} \bar{P}_{i+1}(s, x), \quad K \leqq i \leqq N-1} \\
& {\left[s+\partial / \partial x+\mu_{0}(x)\right] \bar{P}_{K-1}(s, x)=K \lambda_{0} \bar{P}_{K}(s, x)} \\
& {\left[s+\partial / \partial x+\mu_{j}(x)\right] \bar{P}_{i j}(s, x)=0, \quad K \leqq i \leqq N-1, \quad 1 \leqq j \leqq M} \\
& {\left[s+\partial / \partial x+\mu_{m+M}^{*}(x)\right] \bar{Q}_{0}(s, x)=0} \\
& \quad \bar{P}_{i}(s, 0)=\delta_{i, N-1} N \lambda_{0} \bar{P}_{N}(s)+\int_{0}^{\infty} \mu_{0}(x) \bar{P}_{i-1}(s, x) d x+\sum_{j=1}^{M} \int_{0}^{\infty} \mu_{j}(x) \bar{P}_{i j}(s, x) d x \\
& \quad+\delta_{i, m} \int_{0}^{\infty} \mu_{m+M}^{*}(x) \bar{Q}_{0}(s, x) d x, \quad K \leqq i \leqq N-1 \\
& \bar{P}_{K-1}(s, 0)=0, \\
& P_{i j}(s, 0)=\lambda_{j} \bar{P}_{i}(s), \quad K \leqq i \leqq N-1, \quad 1 \leqq j \leqq M \\
& \bar{Q}_{0}(s, 0)=\lambda^{*} \sum_{i=K}^{N} \bar{P}_{i}(s)
\end{aligned}
$$

From (A.4), (A.5), (A.8), (A.9) and (A.1) we obtain

$$
\begin{aligned}
& \bar{P}_{i j}(s, x)=\lambda_{j} \bar{P}_{i}(s) \exp \left[-s x-\int_{0}^{x} \mu_{j}(t) d t\right], \quad K \leqq i \leqq N-1, \quad 1 \leqq j \leqq M, \\
& \bar{Q}_{0}(s, x)=\lambda^{*} \sum_{i=K}^{N} \bar{P}_{i}(s) \exp \left[-s x-\int_{0}^{x} \mu_{m+M}^{*}(z) d z\right] \\
& \alpha\left(s, N, \lambda^{*}\right) \bar{P}_{N}(s)=\int_{0}^{\infty} \mu_{0}(x) \bar{P}_{N-1}(s, x) d x+1 .
\end{aligned}
$$

Using (A.2) and Laplace transform of (3.11), we have

$$
\left[s+\partial / \partial x+i \lambda_{0}+\lambda+\lambda^{*}+\mu_{0}(x)\right] \bar{U}_{i}(s, x)=0, K \leqq i \leqq N-1,
$$

which gives

$$
\bar{U}_{i}(s, x)=\bar{U}_{i}(s, 0) \exp \left[-\left(s+i \lambda_{0}+\lambda+\lambda^{*}\right) x-\int_{0}^{x} \mu_{0}(z) d z\right],
$$

From (3.12), (A.14) and (A.3) we obtain 
RELIABILITY AND MAINTAINABILITY OF A MULTICOMPONENT SERIES-PARALLEL SYSTEM

$$
\begin{aligned}
\bar{P}_{i}(s, x)= & \sum_{n=i}^{N-1}(-1)^{n-i}\left(\begin{array}{c}
n \\
i
\end{array}\right) \bar{U}_{n}(s, 0) \exp \left[-\left(s+n \lambda_{0}+\lambda+\lambda^{*}\right) x-\int_{0}^{x} \mu_{0}(z) d z\right], \\
\bar{P}_{K-1}(s, x)= & \exp \left[-\int_{0}^{x}\left(s+\mu_{0}(z)\right) d z\right] \\
& \cdot\left\{\int_{0}^{x} \exp \left[\int_{0}^{x}\left(s+\mu_{0}(z)\right) d z\right] K \lambda_{0} \bar{P}_{K}(s, x) d x+\bar{P}_{K-1}(s, 0)\right\} \\
= & \exp \left[-s x-\int_{0}^{x} \mu_{0}(z) d z\right]_{n=K}^{N-1}(-1)^{n-K}\left(\begin{array}{c}
n \\
K
\end{array}\right) K \lambda_{0} \bar{U}_{n}(s, 0) \\
& \cdot\left\{1-\exp \left[-\left(n \lambda_{0}+\lambda+\lambda^{*}\right) x\right]\right\} /\left[n \lambda_{0}+\lambda+\lambda^{*}\right]
\end{aligned}
$$

Using (A.10)-(A.12), (A.15) and (A.16) and nothing that $\bar{P}_{i}(s)=\int_{0}^{\infty} \bar{P}_{i}(s, x) d x(K-1 \leqq i \leqq N-1)$, $\bar{P}_{i j}(s)=\int_{0}^{\infty} \bar{P}_{i j}(s, x) d x \quad(K \leqq i \leqq N-1,1 \leqq j \leqq M)$ and $\bar{Q}_{0}(s)=\int_{0}^{\infty} \bar{Q}_{0}(s, x) d x$, we obtain (3.13)-(3.

17). Next we will calculate $\bar{U}_{K}(s, 0), \cdots, \bar{U}_{N-1}(s, 0)$ (see reference [6]). From (3.11), (A.6), (3.16), (A.11), (A.10) and intermediate manipulation we obtain

$$
\begin{aligned}
\bar{U}_{i}(s, 0)= & \sum_{n=i}^{N-1}\left(\begin{array}{c}
n \\
i
\end{array}\right) \bar{P}_{n}(s, 0)=\sum_{n=i}^{N-1}\left(\begin{array}{c}
n \\
i
\end{array}\right)\left\{\delta_{n, N-1} N \lambda_{0} \bar{P}_{N}(s)+\int_{0}^{\infty} \mu_{0}(x) \bar{P}_{n-1}(s, x) d x\right. \\
& \left.+\delta_{n, m} \int_{0}^{\infty} \mu_{m+M}^{*}(x) \bar{Q}_{0}(s, x) d x+\sum_{j=1}^{M} \int_{0}^{\infty} \mu_{j}(x) \bar{P}_{n j}(s, x) d x\right\} \\
= & \left(\begin{array}{c}
N-1 \\
i
\end{array}\right) N \lambda_{0}\left\{\left[\bar{f}_{0}\left(s+(N-1) \lambda_{0}+\lambda+\lambda^{*}\right) \bar{U}_{N-1}(s, 0)+1\right] / \alpha\left(s, N, \lambda^{*}\right)\right\} \\
& +\int_{0}^{\infty} \mu_{0}(x) \bar{U}_{i-1}(s, x) d x+\int_{0}^{\infty} \mu_{0}(x) \bar{U}_{i}(s, x) d x-\left(\begin{array}{c}
N \\
i
\end{array}\right) \int_{0}^{\infty} \mu_{0}(x) \bar{U}_{N-1}(s, x) d x \\
& +\left(\begin{array}{c}
m \\
i
\end{array}\right) \bar{g}_{m+M}^{*}(s) \lambda^{*} \sum_{j=K}^{N} \bar{P}_{j}(s)+\lambda \bar{f}(s) \sum_{n=i}^{N-1}\left(\begin{array}{c}
n \\
i
\end{array}\right) \bar{P}_{n}(s) \\
= & \left(\begin{array}{c}
N \\
i
\end{array}\right)\left\{(N-i) \lambda_{0}+\left[(N-i) \lambda_{0}-\alpha\left(s, N, \lambda^{*}\right)\right] \bar{f}_{0}\left(s+(N-1) \lambda_{0}+\lambda+\lambda^{*}\right) \bar{U}_{N-1}(s, 0)\right\} \\
& / \alpha\left(s, N, \lambda^{*}\right)+\bar{f}_{0}\left(s+i \lambda_{0}+\lambda+\lambda^{*}\right) \bar{U}_{i}(s, 0) \\
& +\bar{f}_{0}\left(s+(i-1) \lambda_{0}+\lambda+\lambda^{*}\right) \bar{U}_{i-1}(s, 0)+\lambda \bar{f}(s)\left[\left(1-\bar{f}_{0}\left(s+i \lambda_{0}+\lambda+\lambda^{*}\right)\right)\right. \\
& \left./\left(s+i \lambda_{0}+\lambda+\lambda^{*}\right)\right] \bar{U}_{i}(s, 0)+\left(\begin{array}{c}
m \\
i
\end{array}\right) \lambda^{*} \bar{g}_{m+M}^{*}(s) \sum_{j=K}^{N} \bar{P}_{j}(s)
\end{aligned}
$$

which gives

$$
\begin{aligned}
\left\{1-\bar{f}_{0}(s+\right. & \left.\left.i \lambda_{0}+\lambda+\lambda^{*}\right)-\lambda \bar{f}(s)\left[\left(1-\bar{f}_{0}\left(s+i \lambda_{0}+\lambda+\lambda^{*}\right)\right) /\left(s+i \lambda_{0}+\lambda+\lambda^{*}\right)\right] \bar{U}_{i}(s, 0)\right\} \\
= & \bar{f}_{0}\left(s+(i-1) \lambda_{0}+\lambda+\lambda^{*}\right) \bar{U}_{i-1}(s, 0)+\left(\begin{array}{c}
m \\
i
\end{array}\right) \lambda^{*} \bar{g}_{m+M}^{*}(s) \sum_{j=K}^{N} \bar{P}_{j}(s) \\
& +\left(\begin{array}{c}
N \\
i
\end{array}\right)\left[(N-i) \lambda_{0}-\alpha\left(s, i, \lambda^{*}\right) \bar{f}_{0}\left(s+(N-1) \lambda_{0}+\lambda+\lambda^{*}\right)\right. \\
& \left.\cdot \bar{U}_{N-1}(s, 0)\right] / \alpha\left(s, N, \lambda^{*}\right), \quad K+1 \leqq i \leqq N-1
\end{aligned}
$$

Multiplying (A.18) by $V_{i}^{(1)}(s)$ and adding for $i=K+1, K+2, \cdots, i$, we obtain

$$
\begin{gathered}
\bar{f}_{0}\left(s+i \lambda_{0}+\lambda+\lambda^{*}\right) V_{i}^{(1)}(s) \bar{U}_{i}^{(1)}(s, 0) \\
=\bar{f}_{0}\left(s+K \lambda_{0}+\lambda+\lambda^{*}\right) \bar{U}_{K}(s, 0)+\sum_{j=K+1}^{i} V_{j-1}^{(1)}(s)\left\{\left(\begin{array}{c}
m \\
j
\end{array}\right) \lambda^{*}\right. \\
-19-
\end{gathered}
$$




$$
\begin{aligned}
& \cdot \bar{g}_{m+M}^{*}(s) \sum_{\gamma=K}^{N} \bar{P}_{\gamma}(s)+\left(\begin{array}{c}
N \\
j
\end{array}\right)(N-j) \lambda_{0}-\alpha\left(s, j, \lambda^{*}\right) \\
& \left.\left.\cdot \bar{f}_{0}\left(s+(N-1) \lambda_{0}+\lambda+\lambda^{*}\right) \bar{U}_{N-1}(s, 0)\right] / \alpha\left(s, N, \lambda^{*}\right)\right\}
\end{aligned}
$$

Setting $i=N-1$, we have

$$
\begin{aligned}
\sum_{j=K+1}^{N}\left(\begin{array}{c}
N \\
j
\end{array}\right)\left[\alpha\left(s, j, \lambda^{*}\right) / \alpha\left(s, N, \lambda^{*}\right)\right] V_{j-1}^{(1)}(s) \bar{f}_{0}\left(s+(N-1) \lambda_{0}+\lambda+\lambda^{*}\right) \bar{U}_{N-1}(s, 0) \\
=\bar{f}_{0}\left(s+K \lambda_{0}+\lambda+\lambda^{*}\right) \bar{U}_{K}(s, 0)+\sum_{j=K+1}^{N-1}\left(\begin{array}{c}
N \\
j
\end{array}\right)(N-j) \lambda_{0} V_{j-1}^{(1)}(s) / \alpha\left(s, N, \lambda^{*}\right) \\
+\lambda^{*} \bar{g}_{m+M}^{*}(s) \sum_{j=K}^{N} \bar{P}_{j}(s) \sum_{j=K+1}^{N-1}\left(\begin{array}{c}
m \\
j
\end{array}\right) V_{j-1}^{(1)}(s)
\end{aligned}
$$

which gives

$$
\bar{U}_{N-1}(s, 0)=a_{N-1}^{(1)}(s) \bar{U}_{K}(s, 0)+\lambda^{*} \bar{g}_{m+M}(s) \sum_{j=K}^{N} \bar{P}_{j}(s) b_{N-1}^{(1)}(s)+b_{N-1}^{(1) *}(s)
$$

Substituting (A.21) into (A.19), we obtain after some manipulation and simplification

$$
\bar{U}_{i}(s, 0)=a_{i}^{(1)}(s) \bar{U}_{K}(s, 0)+\lambda^{*} \bar{g}_{m+M}^{*}(s) \sum_{j=K}^{N} \bar{P}_{j}(s) b_{i}^{(1)}(s)+b_{i}^{(1) *}(s), \quad K+1 \leqq i \leqq N-1,
$$

Substituting (A.22) into (3.15) and (3.16) and arranging for $\sum_{j=K}^{N} \bar{P}_{j}(s)$ and nothing that

$$
\sum_{i=K}^{N-1} \sum_{n=i}^{N-1}(-1)^{n-i}\left(\begin{array}{c}
n \\
i
\end{array}\right) f_{n}(x)=\sum_{n=K}^{N-1}(-1)^{n-K}\left(\begin{array}{c}
n-1 \\
K-1
\end{array}\right) f_{n}(x),
$$

, we obtain (3.20).

Substituting (3.20) into (A.22) we have (3.18).

Since

$$
\sum_{n=i}^{m}(-1)^{n}\left(\begin{array}{c}
m \\
n
\end{array}\right)\left(\begin{array}{l}
n \\
i
\end{array}\right)= \begin{cases}(-1)^{i}, & m=i \\
0, & m \neq i\end{cases}
$$

we have

$$
\begin{aligned}
& \int_{0}^{\infty} \mu_{0}(x) \sum_{n=K}^{N-1}\left(\begin{array}{l}
n \\
K
\end{array}\right) \bar{P}_{n-1}(s, x) d x \\
&=\int_{0}^{\infty} \mu_{0}(x)\left[\bar{P}_{K-1}(s, x)+\sum_{n=K+1}^{N-1}\left(\begin{array}{l}
n \\
K
\end{array}\right) \bar{P}_{n-1}(s, x)\right] d x \\
&=\int_{0}^{\infty} \mu_{0}(x) \bar{P}_{K-1}(s, x) d x+\int_{0}^{\infty} \mu_{0}(x) \sum_{r=K}^{N-1}\left(\begin{array}{c}
r+1 \\
K
\end{array}\right) \bar{P}_{r}(s, x) d x \\
&-\left(\begin{array}{l}
N \\
K
\end{array}\right) \int_{0}^{\infty} \mu_{0}(x) \bar{P}_{N-1}(s, x) d x \\
&= \int_{0}^{\infty} \mu_{0}(x) \bar{P}_{K-1}(s, x) d x+\int_{0}^{\infty} \mu_{0}(x) \bar{U}_{K}(s, x) d x \\
&+\sum_{r=K}^{N-1}\left(\begin{array}{c}
r \\
K-1
\end{array}\right) \int_{0}^{\infty} \mu_{0}(x) \sum_{n=r}^{N-1}(-1)^{n-r}\left(\begin{array}{l}
n \\
r
\end{array}\right) \bar{U}_{n}(s, x) d x-\left(\begin{array}{l}
N \\
K
\end{array}\right) \int_{0}^{\infty} \mu_{0}(x) \bar{U}_{N-1}(s, x) d x \\
&= \sum_{n=K}^{N-1}(-1)^{n-K}\left(\begin{array}{l}
n \\
K
\end{array}\right) K \lambda_{0} \bar{U}_{n}(s, 0)\left[\bar{f}_{0}(s)-\bar{f}_{0}\left(s+n \lambda_{0}+\lambda+\lambda^{*}\right)\right] /\left(n \lambda_{0}+\lambda+\lambda^{*}\right)
\end{aligned}
$$


RELIABILITY AND MAINTAINABILITY OF A MULTICOMPONENT SERIES-PARALLEL SYSTEM

$$
\begin{aligned}
& +\bar{f}_{0}\left(s+K \lambda_{0}+\lambda+\lambda^{*}\right) \bar{U}_{K}(s, 0)+\sum_{n=K}^{N-1}(-1)^{n-K}\left(\begin{array}{c}
n \\
K-1
\end{array}\right) \bar{f}_{0}\left(s+n \lambda_{0}+\lambda+\lambda^{*}\right) U_{n}(s, 0) \\
& -\left(\begin{array}{l}
N \\
K
\end{array}\right) \bar{f}_{0}\left(s+(N-1) \lambda_{0}+\lambda+\lambda^{*}\right) \bar{U}_{N-1}(s, o),
\end{aligned}
$$

Noting that (3.11) and (3.12) do not define for $i=K-1$, we obtain by using arguments analogous to those in the calculation of (A.22),

$$
\begin{aligned}
\bar{U}_{K}(s, 0)= & \sum_{n=K}^{N-1}\left(\begin{array}{l}
n \\
K
\end{array}\right) \bar{P}_{n}(s, 0) \\
= & \left(\begin{array}{c}
N-1 \\
K
\end{array}\right) N \lambda_{0} \bar{P}_{N}(s)+\int_{0}^{\infty} \mu_{0}(x) \sum_{n=K}^{N-1}\left(\begin{array}{c}
n \\
K
\end{array}\right) \bar{P}_{n-1}(s, x) d x \\
& +\sum_{j=1}^{M} \int_{0}^{\infty} \mu_{j}(x) \sum_{n=K}^{N-1}\left(\begin{array}{c}
n \\
K
\end{array}\right) \bar{P}_{n j}(s, x) d x+\left(\begin{array}{c}
m \\
K
\end{array}\right) \int_{0}^{\infty} \mu_{m+M}^{*}(x) \bar{Q}_{0}^{*}(s, x) d x \\
= & \left(\begin{array}{c}
N-1 \\
K
\end{array}\right) N \lambda_{0}\left\{\bar{f}_{0}\left(s+(N-1) \lambda_{0}+\lambda+\lambda^{*}\right) \bar{U}_{N-1}(s, 0)+1\right\} / \alpha\left(s, N, \lambda^{*}\right) \\
& +\sum_{n=K}^{N-1}(-1)^{n-K}\left(\begin{array}{c}
n \\
K
\end{array}\right) K \lambda_{0} \bar{U}_{n}(s, 0) \\
& +\left[\bar{f}_{0}(s)-\bar{f}_{0}\left(s+n \lambda_{0}+\lambda+\lambda^{*}\right)\right] /\left(n \lambda_{0}+\lambda+\lambda^{*}\right)+\bar{f}_{0}\left(s+K \lambda_{0}+\lambda+\lambda^{*}\right) \bar{U}_{K}(s, 0) \\
& +\sum_{n=K}^{N-1}(-1)^{n-K}\left(\begin{array}{c}
n \\
K-1
\end{array}\right) \bar{f}_{0}\left(s+n \lambda_{0}+\lambda+\lambda^{*}\right) \bar{U}_{n}(s, 0) \\
& -\left(\begin{array}{c}
N \\
K
\end{array}\right) \bar{f}_{0}\left(s+(N-1) \lambda_{0}+\lambda+\lambda^{*}\right) U_{N-1}(s, 0) \\
& +\lambda \bar{f}(s) \bar{U}_{K}(s, 0)\left(1-\bar{f}_{0}\left(s+K \lambda_{0}+\lambda+\lambda^{*}\right)\right) /\left(s+K \lambda_{0}+\lambda+\lambda^{*}\right) \\
& +\left(\begin{array}{l}
m \\
K
\end{array}\right) \lambda^{*} \bar{g}_{m+M}^{*}(s) \sum_{j=K}^{N} \bar{P}_{j}(s) .
\end{aligned}
$$

Substituting (3.20) and (3.18) into (A.26), we obtain (3.19) after some manipulation and simplication.

\section{Appendix 2}

From (3.24), (3.25), (3.28)-(3.30) and (3.21) we obtain

$$
\begin{aligned}
& P_{i j}(x)=\lambda_{j} P_{i} \exp \left[-\int_{0}^{x} \mu_{j}(y) d y\right], \\
& Q_{0}(x)=\lambda^{*} \sum_{j=K}^{N} P_{j} \exp \left[-\int_{0}^{x} \mu_{m+M}^{*}(y) d y\right], \\
& {\left[N \lambda_{0}+\lambda^{*}\right] P_{N}=\int_{0}^{\infty} \mu_{0}(x) P_{N-1}(x) d x}
\end{aligned}
$$

Since the similar discrete transform

$$
\begin{aligned}
U_{i}(x) & =\sum_{n=i}^{N-1}\left(\begin{array}{c}
n \\
i
\end{array}\right) P_{n}(x), \quad K \leqq i \leqq N-1, \\
P_{i}(x) & =\sum_{n=i}^{N-1}(-1)^{n-i}\left(\begin{array}{c}
n \\
i
\end{array}\right) U_{n}(x), \quad K \leqq i \leqq N^{-1},
\end{aligned}
$$

by (3.22) we get

$$
\left[d / d x+i \lambda_{0}+\lambda+\lambda^{*}+\mu_{0}(x)\right] U_{i}(x)=0, \quad K \leqq i \leqq N-1
$$

which gives 


$$
\begin{array}{r}
\text { 経 済学 研究 第 } 52 \text { 巻第 } 6 \text { 号 } \\
U_{i}(x)=U_{i}(0) \exp \left[-\left(i \lambda_{0}+\lambda+\lambda^{*}\right) x-\int_{0}^{x} \mu_{0}(y) d y\right] .
\end{array}
$$

From (A.31), (A.33), (3.23) and (3.27) we have

$$
\begin{gathered}
P_{i}(x)=\sum_{n=i}^{N-1}(-1)^{n-i}\left(\begin{array}{l}
n \\
i
\end{array}\right) U_{n}(0) \exp \left[-\left(n \lambda_{0}+\lambda+\lambda^{*}\right) x-\int_{0}^{x} \mu_{0}(y) d y\right], \quad K \leqq i \leqq N-1, \\
P_{K-1}(x)=\exp \left[-\int_{0}^{x} \mu_{0}(y) d y\right] \sum_{n=K}^{N-1}\left(\begin{array}{l}
n \\
K
\end{array}\right) K \lambda_{0} U_{n}(0)\left\{1-\exp \left[-\left(n \lambda_{0}+\lambda+\lambda^{*}\right) x\right]\right\} /\left(n \lambda_{0}+\lambda+\lambda^{*}\right)
\end{gathered}
$$

Using (A.27)-(A.29), (A.34) and (A.35) and noting that $P_{i j}=\int_{0}^{\infty} P_{i j}(x) d x,(K \leqq i \leqq N, 1 \leqq j \leqq M)$, $Q_{0}=\int_{0}^{\infty} Q_{0}(x) d x$, and $P_{i}=\int_{0}^{\infty} P_{i}(x) d x, K-1 \leqq i \leqq N-1$, we obtain (3.32)-(3.36).

Next we will calculate $U_{K}(0), \cdots, U_{N-1}(0)$ (see reference[6]). By the similar argument to the case of (A.18) and using (A.30) and (3.26) we get

$$
\begin{aligned}
& \left\{1-\bar{f}_{0}\left(i \lambda_{0}+\lambda+\lambda^{*}\right)-\lambda\left[1-\bar{f}_{0}\left(i \lambda_{0}+\lambda+\lambda^{*}\right)\right] /\left(i \lambda_{0}+\lambda+\lambda^{*}\right)\right\} U_{i}(0) \\
& =\bar{f}_{0}\left((i-1) \lambda_{0}+\lambda+\lambda^{*}\right) U_{i-1}(0) \\
& \quad-\left(i \lambda_{0}+\lambda^{*}\right)\left(\begin{array}{c}
N \\
i
\end{array}\right) \bar{f}_{0}\left((N-1) \lambda_{0}+\lambda+\lambda^{*}\right) U_{N-1}(0) /\left(N \lambda_{0}+\lambda^{*}\right)+\lambda^{*}\left(\begin{array}{c}
m \\
i
\end{array}\right) \sum_{j=K}^{N} P_{j}, \\
& K+1 \leqq i \leqq N-1
\end{aligned}
$$

Multiplying (A.36) by $V_{i-1}^{(1)}(0)$ and adding for $i=K+1, K+2, \cdots, i$, we obtain

$$
\begin{gathered}
\bar{f}_{o}\left(i \lambda_{0}+\lambda+\lambda^{*}\right) V_{i}^{(1)}(0) U_{i}(0)=\bar{f}_{0}\left(K \lambda_{o}+\lambda+\lambda^{*}\right) U_{K}(0)+\lambda^{*} \sum_{n=K}^{N} P_{n} \sum_{j=K+1}^{i}\left(\begin{array}{c}
m \\
j
\end{array}\right) V_{j-1}^{(1)}(0) \\
-\left[\bar{f}_{0}\left((N-1) \lambda_{0}+\lambda+\lambda^{*}\right) U_{N-1}(0) /\left(N \lambda_{0}+\lambda^{*}\right)\right] \sum_{j=K+1}^{i}\left(\begin{array}{c}
N \\
j
\end{array}\right)\left(j \lambda_{0}+\lambda^{*}\right) V_{j-1}^{(1)}(0),
\end{gathered}
$$

and setting $i=N-1$ we obtain after some manipulation

$$
U_{N-1}(0)=a_{N-1}^{(1)}(0) U_{K}(0)+\lambda^{*} \sum_{j=K}^{N} P_{j} b_{N-1}^{(1)}(0)
$$

Substituting (A.38) into (A.37), we have

$$
U_{i}(0)=a_{i}^{(1)}(0) U_{K}(0)+\lambda^{*} \sum_{j=K}^{N} P_{j} b_{i}^{(1)}(0), \quad K \leqq i \leqq N-1
$$

Using (3.34), (3.35), (A.39) and (A.23) we obtain

$$
\begin{aligned}
& \sum_{j=K}^{N} P_{i}= \bar{f}_{o}\left((N-1) \lambda_{0}+\lambda+\lambda^{*}\right)\left[a_{N-1}^{(1)}(0) U_{K}(0)+\lambda^{*} b_{N-1}^{(1)}(0) \sum_{i=K}^{N} P_{i}\right] /\left(N \lambda_{0}+\lambda^{*}\right) \\
&+\sum_{i=K}^{N-1} \sum_{n=i}^{N-1}(-1)^{n-i}\left(\begin{array}{c}
n \\
i
\end{array}\right)\left[a_{n}^{(1)}(0) U_{K}(0)\right. \\
&\left.+\lambda^{*} b_{n}^{(1)}(0) \sum_{i=K}^{N} P_{i}\right]\left[1-\bar{f}_{0}\left(n \lambda_{0}+\lambda+\lambda^{*}\right)\right] /\left(n \lambda_{0}+\lambda+\lambda^{*}\right) \\
&= {\left[\bar{f}_{0}\left((N-1) \lambda_{0}+\lambda+\lambda^{*}\right) a_{N-1}^{(1)}(0) /\left(N \lambda_{0}+\lambda^{*}\right)\right.} \\
&\left.+\sum_{n=k}^{N-1}(-1)^{n-K}\left(\begin{array}{c}
n-1 \\
K-1
\end{array}\right) a_{n}^{(1)}(0)\left(1-\bar{f}_{0}\left(n \lambda_{0}+\lambda+\lambda^{*}\right)\right) /\left(n \lambda_{0}+\lambda+\lambda^{*}\right)\right] U_{K}(0) \\
&+\sum_{i=K}^{N} P_{i}\left[\lambda^{*} \bar{f}_{0}\left((N-1) \lambda_{0}+\lambda+\lambda^{*}\right) b_{N-1}^{(1)}(0) /\left(N \lambda_{0}+\lambda^{*}\right)\right. \\
&-22-
\end{aligned}
$$




$$
\left.+\lambda^{*} \sum_{n=K}^{N-1}(-1)^{n-K}\left(\begin{array}{c}
n-1 \\
K-1
\end{array}\right) b_{n}^{(1)}(0)\left(1-\bar{f}_{0}\left(n \lambda_{0}+\lambda+\lambda^{*}\right)\right) /\left(n \lambda_{0}+\lambda+\lambda^{*}\right)\right]
$$

which gives (3.39).

Substituting (3.39) into (A. 39) we have (3.27). From (3.81)-(3.36), (3.37) and (3.39) we get

$$
\begin{aligned}
1= & \sum_{n=K}^{N} P_{n}+P_{K-1}+P_{N} \sum_{j=1}^{M} \lambda_{j} K_{j}+\sum_{j=1}^{M} \lambda_{j} K_{j} \sum_{i=K}^{N-1} P_{i}+\lambda^{*} K_{m+M}^{*} \sum_{i=K}^{N} P_{i} \\
= & k_{1}^{(1)}(0)\left[1+\lambda^{*} K_{m+M}^{*}+\sum_{j=1}^{M} \lambda_{j} K_{j}\right] U_{K}(0)+\sum_{n=K}^{N-1}(-1)^{n-K}\left(\begin{array}{c}
n \\
K
\end{array}\right)\left[K \lambda_{0} /\left(n \lambda_{0}+\lambda+\lambda^{*}\right)\right] \\
& \cdot\left[K_{0}-\left(1-\bar{f}_{0}\left(n \lambda_{0}+\lambda+\lambda^{*}\right)\right) /\left(n \lambda_{0}+\lambda+\lambda^{*}\right)\right] a_{n}^{(1) \prime}(0) U_{K}(0)
\end{aligned}
$$

which gives (3.38).

\section{REFERENCES}

[1] D. K. Kulshrestha, "Reliability of a parallel redundant complex system," Opns. Res. 16, No. 1, 1968.

[2] D. K. Kulshrestha, "Reliability of a repairable multicomponent system with redundancy in parallel," IEEE. Trans. on Reliability. 19, No. 2, 1970.

[3] H. Nakamichi, J. Fukuta, S. Takamatsu, and M. Kodama, "Reliability consideration on a repairable multicomponent system with redundancy in parallel," J. Opns. Res. Soc. of Japan. 17, No. 1, 1974.

[4] M. Kodama, "Probabilistic analysis of a multicomponent seriesparallel system under preemptive repeat repair discipline," Opns. Res. 24, No. 23, 1976.

[5] M. Kodama and I. Sawa, "Reliability and maintainability of a multicomponent series-parallel system under several repair disciplines," Microelectron. . Reliab. 22, No. 6, 1982.

[6] M. Kodama and I. Sawa, "Reliability and maintainability of a multicomponent series-parallel system with simultaneous failure and repair priorities," to be published in Microelectron. Reliab.

[7] M. Kodama, "Reliability and maintainability of a multicomponent series-parallel system I," Society of Political Economy, Kyushu University, Vol 46, Nos. 1-2, 1981 (in Japanese).

[8] M. Kodama, "Reliability and maintainability of a multicomponent series-parallel system II," Society of Political Economy, Kyushu University, Vol. 46, Nos. 4-5, 1981 (in Japanese).

[9] M. Kodama and K. Adachi, "On the steady state availability of a single-sever k-out-of-n: G system with simultaneous failure and general repair distribution," Bulletin of Nagoya Institute of Technology, Vol. 30, 1978.

[10] N. K. Jaiswal, Priority Queues, Academic Press, New York, 1968. 